\title{
Motor cortex excitability and inhibitory imbalance in autism spectrum disorder assessed with transcranial magnetic stimulation: a systematic review
}

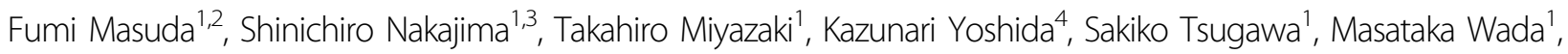 \\ Kamiyu Ogyu ${ }^{1}$, Paul E. Croarkin ${ }^{5}$, Daniel M. Blumberger ${ }^{6}$, Zafiris J. Daskalakis ${ }^{6}$, Masaru Mimura $^{1}$ and Yoshihiro Noda (1)
}

\begin{abstract}
Cortical excitation/inhibition (E/I) imbalances contribute to various clinical symptoms observed in autism spectrum disorder (ASD). However, the detailed pathophysiologic underpinning of E/I imbalance remains uncertain. Transcranial magnetic stimulation (TMS) motor-evoked potentials (MEP) are a non-invasive tool for examining cortical inhibition in ASD. Here, we conducted a systematic review on TMS neurophysiology in motor cortex (M1) such as MEPs and shortinterval intracortical inhibition (SICl) between individuals with ASD and controls. Out of 538 initial records, we identified six articles. Five studies measured MEP, where four studies measured SICI. There were no differences in MEP amplitudes between the two groups, whereas SICI was likely to be reduced in individuals with ASD compared with controls. Notably, SICl largely reflects GABA(A) receptor-mediated function. Conversely, other magnetic resonance spectroscopy and postmortem methodologies assess GABA levels. The present review demonstrated that there may be neurophysiological deficits in GABA receptor-mediated function in ASD. In conclusion, reduced GABAergic function in the neural circuits could underlie the E/I imbalance in ASD, which may be related to the pathophysiology of clinical symptoms of ASD. Therefore, a novel treatment that targets the neural circuits related to GABA(A) receptor-mediated function in regions involved in the pathophysiology of ASD may be promising.
\end{abstract}

\section{Introduction}

Autism spectrum disorder (ASD) is a neurodevelopmental disorder with impairments in social interaction and communication, accompanied with restricted and repetitive behaviors ${ }^{1}$. Although little is known about the neurobiological mechanisms underlying the symptoms of ASD, impaired cortical inhibitory control might explain some of the symptoms. Previous studies have shown that the E/I imbalance could impair sensory, mnemonic, social, emotional, and other forms of neurocognitive

\footnotetext{
Correspondence: Yoshihiro Noda (yoshi-tms@keio.jp)

'Department of Neuropsychiatry, Keio University School of Medicine, Tokyo, Japan

${ }^{2}$ Department of Psychiatry, Shiga University of Medical Science, Shiga, Japan Full list of author information is available at the end of the article.
}

function, depending on the affected neural network ${ }^{2,3}$. As these symptoms are commonly found in ASD, impaired cortical inhibition could explain the pathophysiology of this neurodevelopmental disorder ${ }^{4-6}$. For example, recent studies reported reduced inhibitory control in a Go-NoGo task in adults with ASD compared with controls ${ }^{7-9}$.

Specifically, over $90 \%$ of individuals with ASD display more than one type of sensory dysfunction with extreme levels such as hypersensitivity ${ }^{10}$, which may stem from the inhibitory dysfunction on sensory inputs. Furthermore, impaired inhibition might also account for some of the difficulties in social communication in ASD such as "aloof", "passive", and "active-but-odd" attitudes ${ }^{11,12}$. For example, children with "active-but-odd" characteristics tend to seek interactions with others actively, albeit in an 
unusual way ${ }^{13}$, possibly owing to an neurophysiological excitation/inhibition (E/I) imbalance and manifestation of clinical symptoms such as sensory deficits and/or social communication deficits in individuals with ASD ${ }^{14-16}$.

The E/I balance is assumed to be continuously controlled by the interaction of gamma-aminobutyric acid (GABA)ergic and glutamatergic functions. Especially, GABA receptor-mediated dysfunction has been proposed as a pathological hypothesis in ASD, which posits that the clinical symptoms including a deterioration in the quality of sensory information may be caused by the failure to suppress competing "noise"17. Indeed, prepulse inhibition, involving the sensorimotor gating through GABAergic neurotransmission ${ }^{18,19}$, is impaired in adults with $\mathrm{ASD}^{20,21}$. Furthermore, previous studies showed decreased gamma-band oscillation activity in the temporal area evoked by a pure tone in individuals with ASD compared with healthy controls ${ }^{22,23}$, which may represent reduced activity of inhibitory GABAergic interneurons in $\mathrm{ASD}^{24,25}$.

From a neurochemical point of view, magnetic resonance spectroscopy (MRS) studies showed reduced GABA levels in the anterior cingulate cortex, left auditory cortex, and left motor cortex (M1) of individuals with ASD compared with controls ${ }^{26-30}$. In addition, reduced GABA levels in the anterior cingulate cortex in individuals with ASD were correlated with greater severity of clinical symptoms, such as social cognition ${ }^{26}$, inference emotional state $^{26}$, motor stereotypies ${ }^{31}$, or selfassessment $^{32}$. In postmortem studies, GABA(A) receptors were reduced in the parietal, cerebellar, and frontal regions in individuals with ASD in comparison with healthy controls ${ }^{33,34}$. Collectively, these findings suggest that dysfunction of GABA receptor in the neural circuits may be associated with the pathophysiology of $\mathrm{ASD}^{2,35,36}$.

However, although these results show static findings of substance level of GABA, there is a limit that it does not clarify the physiological dynamics of GABA. Therefore, transcranial magnetic stimulation (TMS) is a useful technique for overcoming the limit, and it enables the exploration of the dynamic functional properties of neural circuits non-invasively. With this technique, we can differentially examine the function of the neural circuits that involves various neurotransmitter associated with cortical functions at the targeted area in human subjects in health or neurological and psychiatric conditions ${ }^{37}$. A recent systematic review and meta-analysis focused on the excitability of M1 suggested a lower cortical inhibition in individuals with attention deficit/hyperactivity disorders (ADHD), another major class of neurodevelopmental disorder, compared with controls $^{38}$. Thus, TMS may be a promising tool to assess the neurophysiological basis represented by the $\mathrm{E} / \mathrm{I}$ balance of ASD. Indeed, several established paradigms are available in TMS research to assess different properties of the neural circuits, for example, motor-evoked potential (MEP), short- interval intracortical inhibition (SICI), intracortical facilitation (ICF), long-interval intracortical inhibition (LICI), and paired associative stimulation (PAS). MEP reflects the excitability of the membrane potential of pyramidal neurons in $\mathrm{M1}^{39}$. SICI largely reflects GABA(A) receptor-mediated inhibitory function ${ }^{40-42}$. SICI is assumed to be induced by through the conditioning stimulus that activates a low threshold inhibitory system, which in turn suppresses the inhibitory interneurons by hyperpolarizing inhibitory postsynaptic potentials, resulting in an inhibition of the cortical output evoked by a subsequent test stimulus ${ }^{40,42,43}$. On the other hand, ICF is assumed to represent excitatory transmission largely through the glutamatergic $N$-methyl-Daspartate receptor ${ }^{44-46}$, which is thought to be evoked by the summation of excitatory postsynaptic potentials ${ }^{47,48}$.

To date, neurochemical studies by MRS and postmortem brain research suggest the abnormal E/I balance involvement in neural circuits at various brain areas in individuals with $\mathrm{ASD}^{3,33,49}$; however, in terms of the TMS neurophysiology, there is still no clear consensus on whether the E/I balance is biased toward excitation or inhibition in the involved neural circuits in ASD. Therefore, we conducted a systematic review on TMS neurophysiology including MEP, SICI, and ICF to identify whether the E/I balance is altered in the involved areas of brain in individuals with ASD.

\section{Methods}

\section{Search strategy}

This systematic review was performed according to the Preferred Reporting Items for Systematic Reviews and MetaAnalyses (PRISMA) guidelines ${ }^{50}$. Electronic databases (PubMed, Medline, Embase, and PsycINFO) were searched up to 27th April 2018 using the terms related to stimulation to the cortex ("non-invasive brain stimulation", "TMS", and "transcranial magnetic stimulation"), those related to assessment of neurophysiology ("brain activity", "brain waves", "EEG", "electrocorticography", "electroencephalogram(s)", "electroencephalography", "EMG", "magnetoencephalography", "MEG", "MEP", "motor-evoked potential", "neurophysiolo", "neuroplasticity", "plasticity", and "plastic"), and those related to neurodevelopmental disorders especially ASD ("ADHD", "ASD", "asperger", "attention deficit hyperactivity disorder", "autism", "autistic", "childhood schizophrenia", "developmental disorder", "neurodevelopmental disability", "neurodevelopmental disorder", and "PDD"). We included "ADHD" as one of search terms, as individuals with ASD often have comorbid diagnosis of ADHD. Two authors (F.M. and Y.N.) independently searched and assessed eligibility, and further extracted data.

\section{Study selection}

Studies were included if articles met the following inclusion criteria: (i) written in English; (ii) involved 
participants diagnosed with ASD by DSM-IV, DSM-IVTR, or ADOS-2 and controls; and (iii) measured with TMS neurophysiology such as MEP and SICI. In contrast, articles were excluded according to the following criteria: (iv) animal model studies; (v) review articles; or (vi) case reports.

\section{Outcome measures}

The outcome measures were TMS neurophysiology regarding MEP, SICI, ICF, LICI, and PAS.

\section{Quality assessment}

The Risk of Bias Assessment tool for Nonrandomized Studies was employed ${ }^{51}$ for the following factors: participant selection, confounding variables, measurement of exposure, blinding of outcome assessment, incomplete outcome data, and selective outcome reporting.

\section{Data extraction}

Data relevant to the research purpose were extracted from each article as follows: (1) characteristics of participants; (2) outcome measures; (3) main results; and (4) mean values and standard deviations for the different excitability parameters. If there was a possibility that the subjects were duplicated among the extracted studies, we asked the author and confirmed the presence or absence of duplication of the subjects. In the present study, we performed preliminary meta-analyses on the above TMS neurophysiological indices and summarized them as supplementary materials.

\section{Results}

The selection process in this systematic review is shown in Fig. 1. The search identified 538 articles, six of which met eligibility criteria. All of the included studies are summarized in Table 1 with extracted data such as study population, investigated measures, and each value of parameters. Among them, five studies measured $\mathrm{MEP}^{4,52-55}$, four studies measured $\mathrm{SICI}^{4,53,54,56}$, two studies measured $\mathrm{LICI}^{54,56}$, one study measured $\mathrm{ICF}^{4}$, and one study measured PAS ${ }^{53}$. There were no studies in which subjects clearly overlapped among the extracted studies. Results of quality assessment such as risk of bias are summarized in Supplementary Figure 1. Further, the funnel plots are displayed in Supplementary Figures 4 and 5 .

\section{MEP}

Five out of the six studies measured $\mathrm{MEP}^{4,52-55}$ (Table 1). All of the five studies identified no differences between individuals with ASD (a total of 101 individuals, age: $22.1 \pm 8.8 \mathrm{yrs}$.) and controls (a total of 83 individuals, age: $23.3 \pm 6.9$ yrs.). Two studies investigated MEP on FDI muscle $^{54,56}$, other two studies did on APB muscle ${ }^{4,53}$, and the other one study did both on FDI and APB muscles ${ }^{52}$.

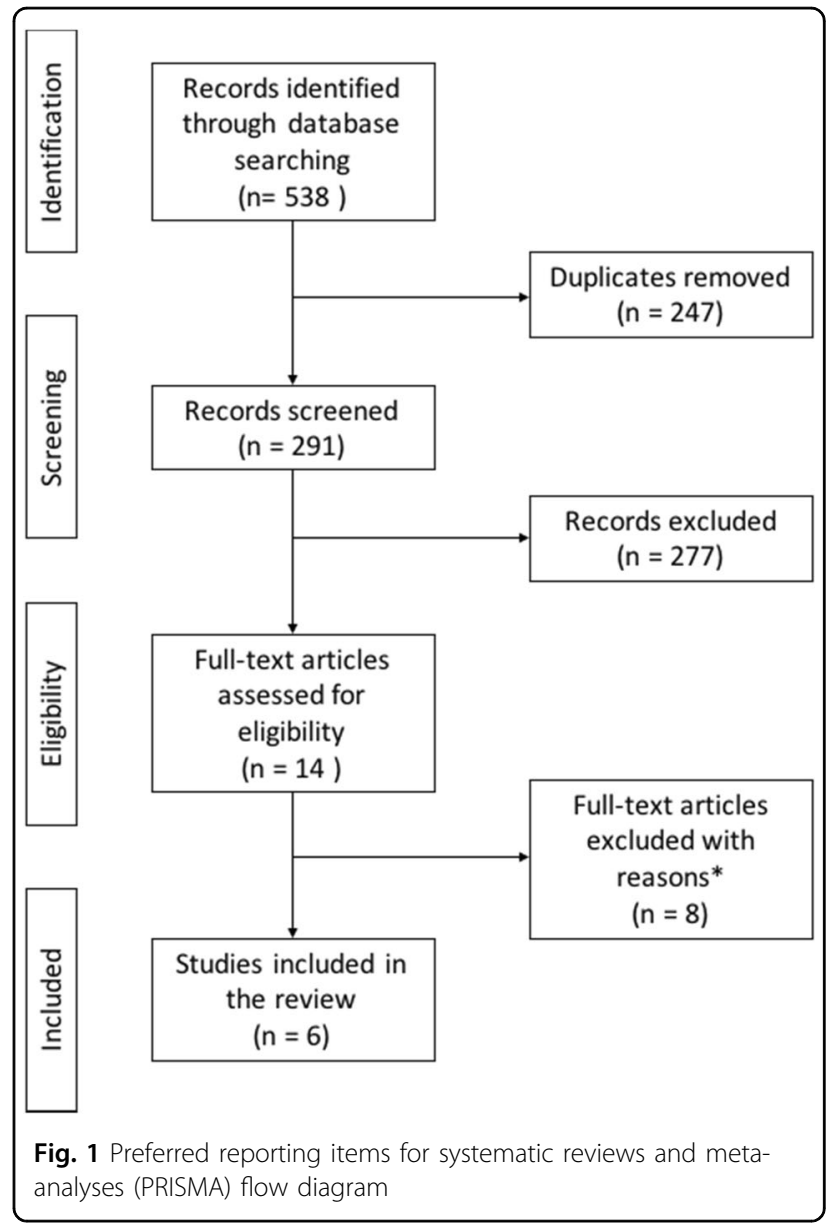

The MEP values measured on FDI muscle is likely to be higher compared with measure on APB.

$\mathrm{SICI}$

Four out of the six studies measured SICI in individuals with ASD (a total of 81 individuals, age: $22.8 \pm$ $10.1 \mathrm{yrs}$.) and controls (a total of 59 individuals, age: $25.3 \pm 8.2$ yrs. $)^{4,53,54,56}$ from the M1 (Table 1). One study showed that individuals with ASD had significantly reduced SICI compared with controls ${ }^{56}$, whereas other three studies showed no differences in SICI between the two groups ${ }^{4,53,54}$. Enticott et al. distinguished autism group to the two group: "high-functioning autism" and "Asperger group". This study showed that SICI was significantly reduced in the highfunctioning autism group compared with both the Asperger disorder group and neurotypical group. There was no significant difference between the Asperger disorder group and neurotypical group. In addition, Enticott et al. ${ }^{54}$ showed ASD with early language delay group had significantly reduced SICI compared with both ASD without language delay group and neurotypical group. 


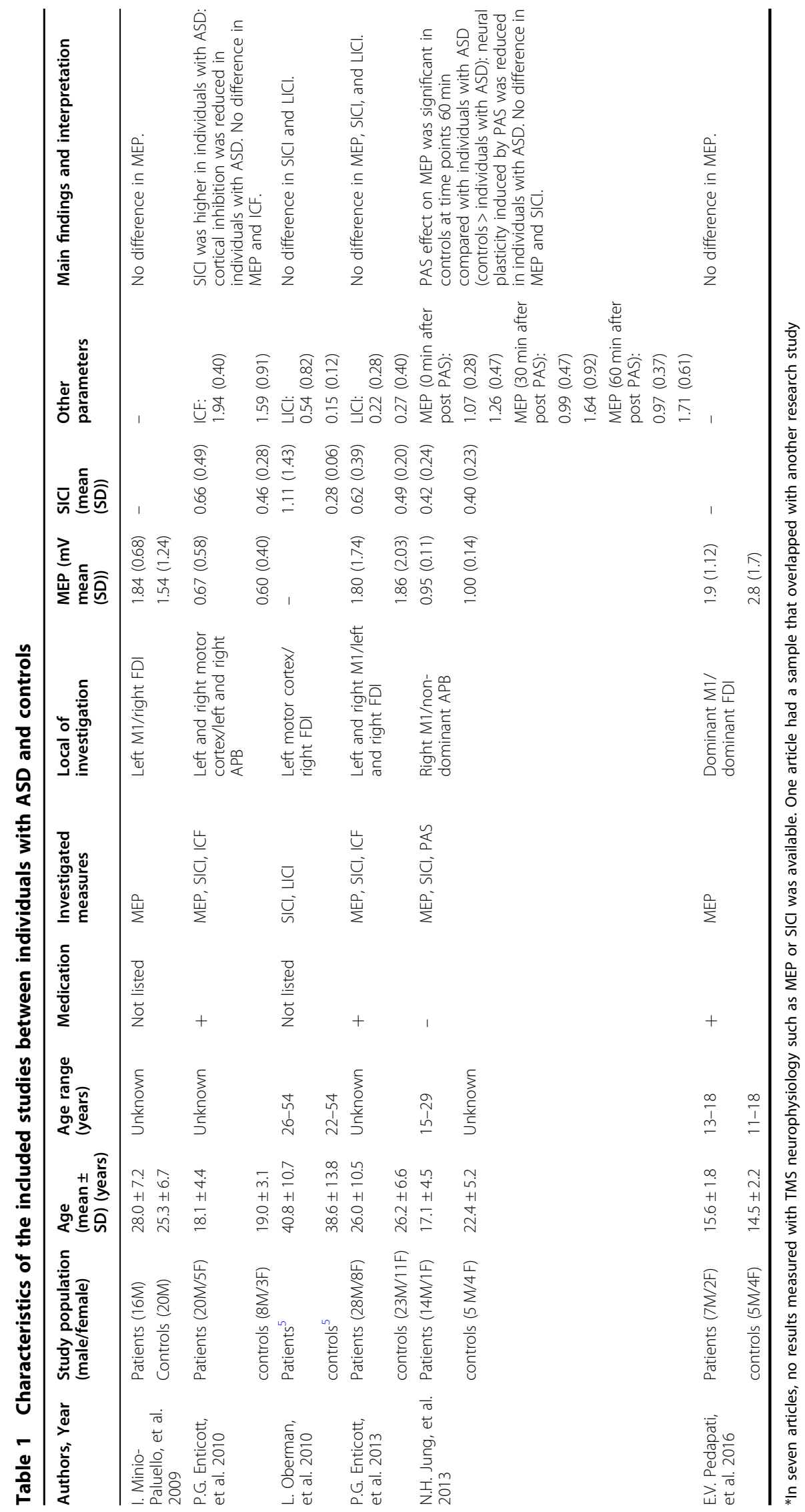




\section{Other TMS metrics of LICl, ICF, and PAS}

Both the two studies on $\mathrm{LICI}^{54,56}$ showed no differences in LICI between the two groups. The one study on $\mathrm{ICF}^{4}$ noted no difference in ICF between the two. Lastly, the one PAS study demonstrated that MEP amplitude in controls was significantly increased at time points of $30 \mathrm{~min}$ and $60 \mathrm{~min}$ after PAS compared with baseline $\mathrm{e}^{53}$, while there was no significant increase in MEP amplitude in individuals with ASD. Although there was no difference in PAS effect on MEP between the two groups at the time point of $30 \mathrm{~min}$, PAS effect on MEP was significant in controls at the time point of $60 \mathrm{~min}$ compared with individuals with ASD (i.e., controls > individuals with ASD).

\section{Discussion}

To our knowledge, this is the first systematic review to compare TMS neurophysiological metrics such as MEP and SICI between individuals with ASD and controls. This systematic review showed that SICI was likely to be reduced in individuals with ASD compared with controls with mild to moderate effect size, whereas there was no difference in MEP amplitude between the two groups.

First, there was no difference in MEP amplitudes between individuals with ASD and controls in each of studies. MEP amplitudes by TMS varied among studies, depending on the muscles of interest. Consistent with previous studies, first dorsal interosseous (FDI) muscle showed relatively higher MEP amplitude, whereas APB muscle showed relatively lower MEP amplitude ${ }^{57,58}$. In ASD, ion channels related to membrane potential such as calcium, sodium, and potassium deficits are to be associated with the underlying pathogenesis contributing to cortical inhibitory dysfunction ${ }^{59}$. Weiss et al ${ }^{60}$ reported that only $5 \%$ of families carried the variants with potential effects on sodium channel function, which mainly relates to membrane potential. In general, it is known that MEP amplitude could be influenced by age, lesion location, motor deficit, and exercises ${ }^{61-63}$. However, the influence of autistic trait on MEP has not been elucidated yet. At least, based on the result of this review, the difference of the membrane potential excitability as indexed by MEP was not observed between the two groups might be masked in individuals with ASD.

Second, as SICI predominantly reflects GABA(A) receptor-mediated function ${ }^{40-42}$, the results of this review suggest that individuals with ASD may have GABA(A) ergic dysfunction, which is consistent with previous stu$\operatorname{dies}^{17,26-28,33,34}$. Furthermore, the degree of cortical inhibition impairment has been shown to be different depending on the subtype of ASD, such as autism with language delay and without delay ${ }^{4,54}$.

In animal studies, mice compromising the phosphodependent regulation of the $\operatorname{GABA}(\mathrm{A})$ receptor $\beta 3$ subunit manifests the core phenotypes of ASD such as increased repetitive behavior and decreased social interaction $^{64}$, whereas mice lacking Mecp2 gene in the GABAreleasing neurons showed increased autistic features such as long interaction with an unfamiliar mouse compared with controls ${ }^{65}$. Indeed, a small percentage of individuals with ASD carry mutations in genes encoding neuroligins, which result in abnormality in postsynaptic cell-adhesion molecules ${ }^{66}$. Also, mice with autism-linked neuroligin-3 R451C mutation show impaired social interactions accompanied by an altered inhibitory synaptic transmission $^{66}$. Further, the mutations in GABA(A) receptor subunit genes (i.e., GABRB3, GABRA5, and GABRQ) were identified in patients with ASD $^{36,67-69}$.

As mentioned above, previous MRS studies showed decreased GABA levels in the anterior cingulate cortex, left auditory cortex, and left $M 1^{26-28,33}$, and postmortem studies showed reduced GABA(A) receptors in individuals with ASD compared with controls ${ }^{33,34}$. In addition, there are several MRS studies that demonstrated the positive relationship between GABA levels and severity of symptoms ${ }^{26,31,32}$. Cochran et al. showed that GABA/Cre levels in the ACC were positively correlated with the score of inference emotional state, intelligent quotient, and severity of social cognitive impairment in the individuals with $\mathrm{ASD}^{26}$. Harris et al. ${ }^{31}$ demonstrated that GABA/Cre levels in the ACC were significantly associated with greater severity of motor stereotypies in the individuals with ASD. Brix et al. ${ }^{32}$ found a significant negative correlation in the ASD group between the scores on the Autism Spectrum Screening Questionnaire (ASSQ) and GABA/Cre levels in the left ACC. Although there is no TMS-EEG study that proved the relationship between dynamic GABAergic function and clinical symptoms in ASD, the relationship would be highly expected in this disorder. Taken together, these findings suggest that GABA(A) receptor function as indexed by TMS-EEG can be a promising indicator of intervention and its modulation may be a novel potential treatment for ASD to improve their clinical symptoms. For example, there are some compounds currently under research that target the specific membrane transport protein, related to GABA(A) receptor-mediated function.

However, it is known that benzodiazepines, which are positive allosteric $\mathrm{GABA}(\mathrm{A})$ receptor modulators, can cause atypical responses such as anxiety, aggression, or hyperarousal in individuals with autism ${ }^{70-72}$. This phenomenon is explained by the chloride homeodynamics theory that high levels of intracellular chloride owing to an immature development of the membrane transport proteins, such as the $\mathrm{Na}-\mathrm{K}-\mathrm{Cl}$ cotransporter (NKCC1) and $\mathrm{K}-\mathrm{Cl}$ cotransporter (KCC2), cause an outward chloride current from the GABA(A) receptor, which results in the alteration of nature of $\mathrm{GABA}(\mathrm{A})$ receptor to excitatory 
function ${ }^{73,74}$. Specifically, in immature neuron, abnormal down-regulation of the $\mathrm{KCC} 2$ as well as an upregulation of selective chloride importer, NKCC1, are observed ${ }^{73,74}$. Therefore, the NKCC1 antagonist, bumetanide, is suggested to have beneficial impact by inducing indirect on the symptoms of $\mathrm{ASD}^{75-77}$. Furthermore, in ASD model mouse, $\alpha 2,3$ subunit-selective positive allosteric modulator L-838417 was effective, whereas $\alpha 1$-subunit-selective drug zolpidem exacerbated social deficits ${ }^{78}$. Thus, pharmacological treatment modifying the function of GABA(A) receptor subunits function may contribute to the improvement of the symptoms of ASD.

On the other hand, glutamate, the neurotransmitter involved in the excitatory component of the E/I balance, is suggested to be increased in individuals with $\mathrm{ASD}^{26,33,79-81}$. Indeed, postmortem studies have discovered the structural and functional changes in both glutamatergic excitatory and GABAergic inhibitory circuits in individuals with $\mathrm{ASD}^{80,81}$. Further, MRS studies have indicated that glutamate levels in striatum, anterior cingulate cortex, left cerebellar, and left frontal cortex were increased in individuals with ASD compared with controls ${ }^{26,33,79}$. Collectively, these results suggested that individuals with ASD may have increased cortical excitation in the involved area of brain. Together with the result of SICI, individuals with ASD may have the E/I balance biased toward excitation, as a result of reduced inhibition and/or increased excitation.

In addition, several genetic mutations have been reported in relation to synaptic formation in individuals with ASD such as NLGNs, SHANKs, and PTEN for dendritic spines of pyramidal cells, and NRXNs and FMRP for parvalbumin interneurons like basket cells and chandelier cells ${ }^{80,82}$. The mutations of these genes could cause impaired synapse formation and synaptic pruning, which may lead to the $\mathrm{E} / \mathrm{I}$ imbalance in $\mathrm{ASD}^{83,84}$. These abnormalities in synaptic proteins may be linked to the E/ I imbalance in individuals with ASD via altered neural system that involved GABA receptor-mediated function as suggested by the SICI studies on ASD.

However, there are several studies that are inconsistent with previously determined direction of possible E/I balance bias in $\mathrm{ASD}^{85-88}$. Contrary to our finding, several MRS studies reported that decreased excitation in the frontal lobe, occipital lobe, and anterior cingulate cortex in individuals with ASD compared with controls ${ }^{33,85,86}$. Further, perceptual measures as assessed by the color discrimination or pure pitch discrimination showed increased inhibition in individuals with $\mathrm{ASD}^{87,88}$. In clinical practice, altered responsiveness to sensory input toward both the opposite directions are assessed as either hyposensitivity as known as "low registration" or hypersensitivity as "sensory sensitivity" 89 . Although these symptoms look inconsistent, they often co-exist. Therefore, the assessment of $\mathrm{E} / \mathrm{I}$ balance from different brain regions may be needed to explain the inconsistent symptoms of ASD. Therefore, the present findings warrant further TMS neurophysiological studies with larger sample sizes that include other paradigms such as ICF, LICI, short-latency afferent inhibition, and PAS to elucidate comprehensive understanding of the neural basis of ASD.

\section{Limitation}

The limitation of this systematic review is the small sample size for each parameter. Also, each study included a limited number of individuals, which did not allow us to control the confounding effects of the age, gender, accompanied medication, or comorbidity such as other neurodevelopmental disorders. Further, in this study, because of the limited number of articles investigating the non-motor areas in ASD, only M1 could be systematically reviewed.

\section{Future direction}

Further well-designed studies addressing current limitations and the knowledge gap are warranted. At present, our knowledge is quite limited about the neurobiological mechanism of ASD, although it is the key for establishing methods for precise diagnosis and efficient treatment ${ }^{90}$. Among various paradigms in TMS study, PAS paradigm might be the promising paradigm to seek for. Although we found only one study that measured PAS in this review, PAS paradigm could index the degree of neuroplasticity in motor as well as non-motor areas in individuals with ASD, and thus be useful in assessing the E/I balance in the neural circuits at the targeted brain area. Therapeutic intervention with brain stimulation might also prove beneficial as a novel treatment tool for ASD, for example, by applying repetitive TMS or theta-burst stimulation to readjust $E / I$ balance in specific brain areas ${ }^{91,92}$. By combining the two aspects of TMS as a tool to probe and modulate the neural function, it may even become possible to implement a tailor-made treatment that optimizes therapeutic outcomes in the future, applying appropriate stimulation to the brain regions involved in this disorder.

\section{Conclusions}

This systematic review demonstrated reduced cortical inhibition of M1 in individuals with ASD with no difference of MEP compared with controls. This finding is in line with the converging evidence that suggests altered $\mathrm{E} / \mathrm{I}$ balance in ASD. Given that impaired GABAergic and/or glutamatergic systems could cause the E/I imbalance in the neural circuits, the present systematic review warrant further TMS studies in ASD with larger sample sizes and more-comprehensive neurophysiological and neuropsychological measures. Targeting a wider range of brain areas outside of 


\section{M1-dorsolateral prefrontal cortex-which would be more closely related to cognitive and/or sensory functions of the disorder is the next logical area of investigation.}

\section{Acknowledgements}

This research did not receive any specific grants from funding agencies in the public, commercial, or not-for-profit sectors.

\begin{abstract}
Author details
${ }^{1}$ Department of Neuropsychiatry, Keio University School of Medicine, Tokyo, Japan. ${ }^{2}$ Department of Psychiatry, Shiga University of Medical Science, Shiga, Japan. ${ }^{3}$ Multimodal Imaging Group, Centre for Addiction and Mental Health and Department of Psychiatry, University of Toronto, Toronto, ON, Canada. ${ }^{4}$ Department of Psychiatry and Psychology, Mayo Clinic, Rochester, MN, USA. ${ }^{5}$ Pharmacogenetics Research Clinic, Centre for Addiction and Mental Health, Toronto, ON, Canada. ${ }^{6}$ Temerty Centre for Therapeutic Brain Intervention, Centre for Addiction and Mental Health and Department of Psychiatry, University of Toronto, Toronto, ON, Canada
\end{abstract}

\section{Conflict of interest}

F.M. reports no biomedical interests. S.N. has received fellowship grants from Canadian Institute of Health Research (ClHR), research support from Japan Society for the Promotion of Science (Grant-in-Aid for Young Scientists A, Grants-in-Aid for Scientific Research B, and Grants-in-Aid for Scientific Research C), Japan Society for the Promotion of Science, Japan Agency for Medical Research and development (AMED), Japan Research Foundation for Clinical Pharmacology, Naito Foundation, Uehara Memorial Foundation, Takeda Science Foundation, Daiichi Sankyo Research Program, and Novartis Research Program and manuscript fees or speaker's honoraria from DainipponSumitomo Pharma and Yoshitomi-Yakuhin within the past 3 years. T.M. reports no biomedical interests. K.Y. has received manuscript fees from Sumitomo Dainippon Pharma, fellowship grants from the Japan Research Foundation for Clinical Pharmacology, and consultant fees from Bracket within the past 3 years. S.T., M.W., R.T., and K.O. report no biomedical interests. P.E.C. has received research grant support from Pfizer, Inc., NIMH, the Brain and Behavior Research Foundation, and the Mayo Clinic Foundation. He has served as a site subprincipal or principal investigator (without additional compensation) for Eli Lilly and Co., Forest Laboratories, Inc., Merck \& Co., Inc., and Pfizer, Inc.; has received equipment support from Neuronetics, Inc.; and receives supplies and genotyping services from Assurex Health, Inc. for an investigator-initiated study. He is the primary investigator for a multicenter study funded by Neuronetics, Inc. He is a site investigator for a study funded by NeoSync, Inc. D. M.B. receives research support from the Canadian Institutes of Health Research $(\mathrm{ClHR})$, National Institutes of Health-US $(\mathrm{NIH})$, Weston Brain Institute, Brain Canada, and the Temerty Family through the CAMH Foundation and the Campbell Research Institute. He received research support and in-kind equipment support for an investigator-initiated study from Brainsway Ltd., and he is the site principal investigator for three sponsor-initiated studies for Brainsway Ltd. He received in-kind equipment support from Magventure for this investigator-initiated study. He received medication supplies for an investigator-initiated trial from Indivior. He has served on an advisory board for Janssen. Z.J.D. has received within the last 3 years both research and equipment in-kind support for an investigator-initiated study through Brainsway Ltd. and Magventure. M.M. has received grants or speaker's honoraria from Asahi Kasei Pharma, Astellas Pharmaceutical, Daiichi Sankyo, Dainippon-Sumitomo Pharma, Eisai, Eli Lilly, GlaxoSmithKline, Janssen Pharmaceutical, Meiji-Seika Pharma, Mochida Pharmaceutical, MSD, Novartis Pharma, Otsuka Pharmaceutical, Pfizer, Shionogi, Takeda, Tanabe Mitsubishi Pharma, and Yoshitomi-Yakuhin within 3 years. Y.N. receives a Grant-in-Aid for Young Scientists (KAKENHI), a research grant from Japan Agency for Medical Research and development (AMED). Y.N. receives an investigator-initiated clinical study grant from TEIJIN PHARMA LIMITED. He also receives research grants from Japan Health Foundation, Meiji Yasuda Mental Health Foundation, Mitsui Life Social Welfare Foundation, Takeda Science Foundation, SENSHIN Medical Research Foundation, Health Science Center Foundation, and Daiichi Sankyo Scholarship Donation Program. He has received research supports from Otsuka Pharmaceutical Co., Ltd, Shionogi \& Co., Ltd., and Meiji-Seika Pharma Co., Ltd. He receives equipment-in-kind support for an investigatorinitiated study from Magventure Inc.

\section{Publisher's note}

Springer Nature remains neutral with regard to jurisdictional claims in published maps and institutional affiliations.

Supplementary information accompanies this paper at (https://doi.org/ 10.1038/s41398-019-0444-3).

Received: 9 August 2018 Revised: 2 February 2019 Accepted: 16 February 2019

Published online: 07 March 2019

\section{References}

1. American Psychiatric Association. Diagnostic and statistical manual of mental disorders 5th edn, 2013.

2. Robertson, C. E. \& Baron-Cohen, S. Sensory perception in autism. Nat. Rev. Neurosci. 18, 671-684 (2017).

3. Rubenstein, J. L. R. \& Merzenich, M. M. Model of autism: increased ratio of excitation/inhibition in key neural systems. Genes Brain Behav. 2, 255-267 (2003).

4. Enticott, P. G., Rinehart, N. J., Tonge, B. J., Bradshaw, J. L. \& Fitzgerald, P. B. A preliminary transcranial magnetic stimulation study of cortical inhibition and excitability in high-functioning autism and Asperger disorder. Dev. Med. Child Neurol. 52, e179-e183 (2010).

5. Vattikuti, S. \& Chow, C. C. A computational model for cerebral cortical dysfunction in autism spectrum disorders. Biol. Psychiatry 67, 672-678 (2010).

6. Banerjee, A. et al. Impairment of cortical GABAergic synaptic transmission in an environmental rat model of autism. Int. J. Neuropsychopharmacol. 16, 1309-1318 (2013).

7. Uzefovsky, F., Allison, C., Smith, P. \& Baron-Cohen, S. Brief report: the Go/No-Go task online: inhibitory control deficits in autism in a large sample. J. Autism Dev. Disord. 46, 2774-2779 (2016)

8. Høyland, A. L. et al. Event-related potentials in a cued Go-NoGo task associated with executive functions in adolescents with autism spectrum disorder: a case-control study. Front. Neurosci. 11: 393 (2017).

9. Tye, C. et al. Attention and inhibition in children with ASD, ADHD and comorbid ASD+ADHD: an event-related potential study. Psychol. Med. 44, 1101-1116 (2013).

10. Crane, L., Goddard, L. \& Pring, L. Sensory processing in adults with autism spectrum disorders. Autism 13, 215-228 (2009).

11. Althaus, M., Renk, M., Minderaa, Ru. B. \& Vries, H. D. Initiatory and reactive behaviour in young childrenwith a pervasive developmental disorder: two independent components in communicative behaviourfor distinguishing subgroups. Eur. Child Adolesc. Psychiatry 3, 242-254 (1994).

12. Wing L, Attwood A. Syndromes of autism and atypical development. (eds. Cohen DJ, Donnellan AM) (John Wiley \& Sons, 1987).

13. Scheeren, A. M., Koot, H. M. \& Begeer, S. Social Interaction style of children and adolescents with high-functioning autism spectrum disorder. J. Autism Dev. Disord. 42, 2046-2055 (2012).

14. Smith, J., Rho, J. M. \& Teskey, G. C. Ketogenic diet restores aberrant cortical motor maps and excitation-to-inhibition imbalance in the BTBR mouse model of autism spectrum disorder. Behav. Brain Res. 304, 67-70 (2016).

15. Palmer, C. J., Lawson, R. P., Shankar, S., Clifford, C. W. G. \& Rees, G. Autistic adults show preserved normalisation of sensory responses in gaze processing. Cortex 103, 13-23 (2018)

16. Selimbeyoglu A, et al. Modulation of prefrontal cortex excitation/inhibition balance rescues social behavior in CNTNAP2-deficient mice. Sci. Transl. Med. $\mathbf{9}$, pii: eaah6733 (2017).

17. Hussman, J. P. Suppressed gabaergic inhibition as a common factor in suspected etiologies of autism. J. Autism Dev. Disord. 31: 247-248 (2001).

18. Yeomans, J. S. et al. GABA receptors and prepulse inhibition of acoustic startle in mice and rats. Eur. J. Neurosci. 31, 2053-2061 (2010).

19. Inui, K., Takeuchi, N., Sugiyama, S., Motomura, E. \& Nishihara, M. GABAergic mechanisms involved in the prepulse inhibition of auditory evoked cortical responses in humans. PLOS ONE 13, e0190481 (2018).

20. Perry, W., Minassian, A., Lopez, B., Maron, L. \& Lincoln, A. Sensorimotor gating deficits in adults with autism. Biol. Psychiatry 61, 482-486 (2007).

21. McAlonan, G. M., Daly, E., Kumari, V. Critchley, H. D., Amelsvoort, Tv. \& Suckling, J. Brain anatomy and sensorimotor gating in Asperger's syndrome. Brain 127, 1594-1606 (2002). 
22. Edgar, J. C. et al. Neuromagnetic oscillations predict evoked-response latency delays and core language deficits in autism spectrum disorders. J. Autism Dev. Disord. 45, 395-405 (2015).

23. Rojas, D. C., Maharajh, K., Teale, P. \& Rogers, S. J. Reduced neural synchronization of gamma-band MEG oscillations in first-degree relatives of children with autism. BMC Psychiatry 8, 66 (2008).

24. Baruth, J. M. et al. Low-frequency repetitive transcranial magnetic stimulation modulates evoked-gamma frequency oscillations in autism spectrum disorder. J. Neurother. 14, 179-194 (2010).

25. Buard, I., Rogers, S., Hepburn, S., Kronberg, E. \& Rojas, D. Altered oscillation patterns and connectivity during picture naming in autism. Front. Hum. Neurosci. 7, 742 (2013)

26. Cochran, D. M. et al. Relationship among glutamine, $\gamma$-aminobutyric acid, and social cognition in autism spectrum disorders. J. Child Adolesc. Psychopharmacol. 25, 314-322 (2015).

27. Gaetz, W. et al. GABA estimation in the brains of children on the autism spectrum: measurement precision and regional cortical variation. Neuroimage 86, 1-9 (2014).

28. Rojas, D., Singel, D., Steinmetz, S., Hepburn, S. \& Brown, M. Decreased left perisylvian GABA concentration in children with autism and unaffected siblings. Neuroimage 86, 28-34 (2014).

29. Ajram, L. A. et al. The contribution of $[1 \mathrm{H}]$ magnetic resonance spectroscopy to the study of excitation-inhibition in autism. Prog. Neuropsychopharmacol. Biol. Psychiatry 89, 236-244 (2019).

30. Schur, R. R. et al. Brain GABA levels across psychiatric disorders: a systematic literature review and meta-analysis of (1) H-MRS studies. Hum. Brain. Mapp. 37, 3337-3352 (2016).

31. Harris, A. D. et al. GABA and glutamate in children with primary complex motor stereotypies: an1H-MRS study at 7T. AJNR Am. J. Neuroradiol. 37, 552-557 (2016).

32. Brix, M. et al. Brain MR spectroscopy in autism spectrum disorder - the GABA excitatory/inhibitory imbalance theory revisited. Front. Hum. Neurosci. 22, 365 (2015).

33. Dickinson, A., Jones, M. \& Milne, E. Measuring neural excitation and inhibition in autism: different approaches, different findings and different interpretations. Brain Res. 1648, 277-289 (2016).

34. Fatemi, S. H., Reutiman, T. J., Folsom, T. D. \& Thuras, P. D. GABA(A) receptor downregulation in brains of subjects with autism. J. Autism Dev. Disord. 39, 223-230 (2009).

35. Chen $\mathrm{C}-\mathrm{H}$, et al. Genetic analysis of GABRB3 as a candidate gene of autism spectrum disorders. Mol. Autism. 5, 36 (2014).

36. Piton, A. et al. Analysis of the effects of rare variants on splicing identifies alterations in GABAA receptor genes in autism spectrum disorder individuals. Eur. J. Hum. Genet. 21, 749-756 (2013).

37. Kobayashi, M. \& Pascual-Leone, A. Transcranial magnetic stimulation in neurology. Lancet Neurol. 2, 145-156 (2003).

38. Dutra, T. G., Baltar, A. \& Monte-Silva, K. K. Motor cortex excitability in attentiondeficit hyperactivity disorder (ADHD): a systematic review and meta-analysis. Res. Dev. Disabil. 56, 1-9 (2016).

39. Nielsen, J. F. \& Nørgaard, P. Increased post-exercise facilitation of motor evoked potentials in multiple sclerosis. Clin. Neurophysiol. 113(8), 1295-300 (2002).

40. Ilić, T. V. et al. Short-interval paired-pulse inhibition and facilitation of human motor cortex: the dimension of stimulus intensity. J. Physiol. 545, 153-167 (2002).

41. Noda, $Y$. et al. Characterization of the influence of age on GABA(A) and glutamatergic mediated functions in the dorsolateral prefrontal cortex using paired-pulse TMS-EEG. Aging (Albany NY). 9, 556-572 (2017).

42. Cash, R. F. et al. Characterization of glutamatergic and GABAA-mediated neurotransmission in motor and dorsolateral prefrontal cortex using pairedpulse TMS-EEG. Neuropsychopharmacology 42, 502-511 (2017).

43. Kujirai, T. et al. Corticocortical inhibition in human motor cortex. J. Physiol. 471, 501-519 (1993).

44. Mori, F. et al. Genetic variants of the NMDA receptor influence cortical excitability and plasticity in humans. J. Neurophysiol. 106, 1637-1643 (2011).

45. Schwenkreisa, $P$. et al. Influence of the $x$-methyl-D-aspartate antagonist memantine on human motor cortex excitability. Neurosci. Lett. 270, 137-140 (1999).

46. Ziemann, U., Hallett, M. \& Cohen, L. G. Mechanisms of deafferentation-induced plasticity in human motor cortex. J. Neurosci. 18, 7000-7007 (1998).
47. Liepert, J., Schwenkreis, P., Tegenthoff, M. \& Malin, J. The glutamate antagonist riluzole suppresses intracortical facilitation. J. Neural Transm. Nienna). 104 1207-1214 (1997).

48. Schwenkreis, P. et al. Riluzole suppresses motor cortex facilitation in correlation to its plasma level. A study using transcranial magnetic stimulation. Exp. Brain Res. 135, 293-299 (2000)

49. Uzunova, G., Pallanti, S. \& Hollander, E. Excitatory/inhibitory imbalance in autism spectrum disorders: implications for interventions and therapeutics. World J. Biol. Psychiatry 17, 174-186 (2016).

50. Moher, D., Liberati, A., Tetzlaff, J., Altman, D. G. \& Group, P. Preferred reporting items for systematic reviews and meta-analyses: the PRISMA statement. J. Clin. Epidemiol. 62, 1006-1012 (2009).

51. Kim, S. Y. et al. Testing a tool for assessing the risk of bias for nonrandomized studies showed moderate reliability and promising validity. J. Clin. Epidemiol. 66, 408-414 (2013).

52. Minio-Paluello, I., Baron-Cohen, S., Avenanti, A., Walsh, V. \& Aglioti, S. M. Absence of embodied empathy during pain observation in asperger syndrome. Biol. Psychiatry 65, 55-62 (2009).

53. Jung, N. H. et al. Impaired induction of long-term potentiation-like plasticity in patients with high-functioning autism and Asperger syndrome. Dev. Med. Child Neurol. 55, 83-89 (2013).

54. Enticott, P. G. et al. GABAergic activity in autism spectrum disorders: an investigation of cortical inhibition via transcranial magnetic stimulation. Neuropharmacology 68, 202-209 (2013).

55. Pedapati, E. V. et al. Abnormal cortical plasticity in youth with autism spectrum disorder: a transcranial magnetic stimulation case-control pilot study. J. Child Adolesc. Psychopharmacol. 26, 625-631 (2016).

56. Oberman, L. et al. Transcranial magnetic stimulation provides means to assess cortical plasticity and excitability in humans with fragile $X$ syndrome and autism spectrum disorder. Front. Synaptic Neurosci. 2, 26 (2010).

57. Dileone, M. et al. Enhanced human brain associative plasticity in Costello syndrome. J. Physiol. 588, 3445-3456 (2010).

58. Murakami, T., Sakuma, K., Nomura, T. \& Nakashima, K. Short-interval intracortical inhibition is modulated by high-frequency peripheral mixed nerve stimulation. Neurosci. Lett. 420, 72-75 (2007).

59. Schmunk, G. \& Gargus, J. Channelopathy pathogenesis in autism spectrum disorders. Front. Genet. 4, 222 (2013).

60. Weiss, L. A. et al. Sodium channels SCN1A, SCN2A and SCN3A in familial autism. Mol. Psychiatry 8, 186-194 (2003).

61. Balbi, P., Perretti, A., Sannino, M., Marcantonio, L. \& Santoro, L. Postexercise facilitation of motor evoked potentials following transcranial magnetic stimulation: a study in normal subjects. Muscle Nerve. Muscle Nerve. 25, 448-452 (2002).

62. Kim, G. W. \& Ko, M. H. Facilitation of corticospinal tract excitability by transcranial direct current stimulation combined with voluntary grip exercise. Neurosci. Lett. 548, 181-184 (2013).

63. Chen, $X$. et al. Success rate of motor evoked potentials for intraoperative neurophysiologic monitoring: effects of age, lesion location, and preoperative neurologic deficits. J. Clin. Neurophysiol. 24, 281-285 (2007).

64. Vien, T. N. et al. Compromising the phosphodependent regulation of the GABAAR $\beta 3$ subunit reproduces the core phenotypes of autism spectrum disorders. Proc. Natl. Acad. Sci. 112, 14805-14810 (2015).

65. Chao, H. T. et al. Dysfunction in GABA signalling mediates autism-like stereotypies and Rett syndrome phenotypes. Nature 468, 263-269 (2010).

66. Tabuchi, $K$. et al. A neuroligin-3 mutation implicated in autism increases inhibitory synaptic transmission in mice. Science 318, 71-76 (2007).

67. Fatemi, S. H. et al. Downregulation of GABAA receptor protein subunitsalpha6, beta2, delta, epsilon, gamma2, theta, and rho2 in superior frontal cortex of subjects with autism. J. Autism Dev. Disord. 44, 1833-1845 (2014).

68. McCauley, J. L. et al. A linkage disequilibrium map of the 1-Mb 15q12 GABA(A) receptor subunit cluster and association to autism. Am. J. Med. Genet. B Neuropsychiatr. Genet. 131B, 51-59 (2004).

69. Yoo, H. K., Chung, S., Hong, J. P., Kim, B. N. \& Cho, S. C. Microsatellite marker in gamma - aminobutyric acid - a receptor beta 3 subunit gene and autism spectrum disorders in Korean trios. Yonsei. Med. J. 50, 304-306 (2009).

70. Marrosu, F., Marrosu, G., Rachel, M. G. \& Biggio, G. Paradoxical reactions elicited by diazepam in children with classic autism. Funct. Neurol. 2, 355-361 (1987).

71. Mancuso, C., Tanzi, M. \& Gabay, M. Paradoxical reactions to benzodiazepines: literature review and treatment options. Pharmacotherapy 24, 1177-1185 (2004). 
72. Bruining, $\mathrm{H}$. et al. Paradoxical benzodiazepine response: a rationale for bumetanide in neurodevelopmental disorders? Pediatrics 136, e539-e543 (2015).

73. Fukuda, A., Muramatsu, K. \& Okabe, A. Changes in intracellular Ca2+induced by GABAA receptor activation and reduction in $\mathrm{Cl}$ - gradient in neonatal rat neocortex. J. Neurophysiol. 79, 439-446 (1998).

74. Ben-Ari, Y. The GABA excitatory/inhibitory developmental sequence: a personal journey. Neuroscience 279, 187-219 (2014).

75. Lemonnier, E. et al. A randomised controlled trial of bumetanide in the treatment of autism in children. Transl. Psychiatry 2, e202 (2012).

76. Lemonnier, E. et al. Effects of bumetanide on neurobehavioral function in children and adolescents with autism spectrum disorders. Transl. Psychiatry $\mathbf{7}$, e1056 (2017)

77. Schulte, J. T., Wierenga, C. J. \& Bruining, H. Chloride transporters and GABA polarity in developmental, neurological and psychiatric conditions. Neurosci. Biobehav. Rev. 90, 260-271 (2018).

78. Han, S., Tai, C., Jones, C. J., Scheuer, T. \& Catterall, W. A. Enhancement of inhibitory neurotransmission by GABAA receptors havingalpha2,3-subunits ameliorates behavioral deficits in a mouse model of autism. Neuron 81, 1282-1289 (2014).

79. Hassan, T. H. et al. Blood and brain glutamate levels in children with autistic disorder. Res. Autism Spectr. Disord. 7, 541-548 (2013).

80. Gao, R. \& Penzes, P. Common mechanisms of excitatory and inhibitory imbalance in schizophrenia and autism spectrum disorders. Curr. Mol. Med. 15 146-167 (2015).

81. Chattopadhyaya, B. \& Cristo, G. GABAergic circuit dysfunctions in neurodevelopmental disorders. Front. Psychiatry 3, 51 (2012).
82. Wong, F. K. et al. Pyramidal cell regulation of interneuron survival sculpts cortical networks. Nature 557, 668-673 (2018).

83. Presumey, J., Bialas, A. R. \& Carroll, M. C. Complement system in neura synapse elimination in development and disease. Adv. Immunol. 135, 53-79 (2017).

84. Tsai, N.-P. et al. Multiple autism-linked genes mediate synapse elimination via proteasomal degradation of a synaptic scaffold PSD-95. Cell 151, 1581-1594 (2012).

85. Bernardi, S. et al. In vivo $1 \mathrm{H}$-magnetic resonance spectroscopy study of the attentional networks in autism. Brain Res. 1380, 198-205 (2011).

86. DeVito, T. J. et al. Evidence for cortical dysfunction in autism: a proton magnetic resonance spectroscopic imaging study. Biol. Psychiatry 61, 465-473 (2007).

87. Bonnel, A. et al. Enhanced pure-tone pitch discrimination among persons with autism but not Asperger syndrome. Neuropsychologia 48, 2465-2475 (2010).

88. Franklin, A. et al. Reduced chromatic discrimination in children with autism spectrum disorders. Dev. Sci. 13, 188-200 (2010).

89. Brown C, Dunn W. Adolescent/adult sensory profile. San Antonio, TX, Psychological Corporation. 2002

90. Masuda, F. et al. Clinical effectiveness of repetitive transcranial magnetic stimulation treatment in children and adolescents with neurodevelopmenta disorders: A systematic review. Autism. 1362361318822502 (2019).

91. Dubin, M. J. et al. Elevated prefrontal cortex GABA in patients with major depressive disorder after TMS treatment measured with proton magnetic resonance spectroscopy. J. Psychiatry Neurosci. 41, E37-E45 (2016).

92. Iwabuchi, S. J. et al. Targeted transcranial theta-burst stimulation alters frontoinsular network and prefrontal GABA. Neuroimage 146, 395-403 (2017). 